\title{
Factores motivacionales y desempeño académico en adolescentes
}

Virgelina Castellanos Páez ${ }^{1}$ y Paola Andrea Reyes Parra²

\section{Introducción}

El desempeño académico se establece como un indicador del nivel de aprendizaje, habilidades y conocimientos alcanzados por el estudiante en determinado periodo de tiempo (Jiménez, 2000).

A nivel nacional e internacional, el estudio del desempeño académico es un campo de investigación relevante a nivel educativo, pues identificar los factores que pueden favorecer o dificultar el desempeño académico posibilita el diseño de estrategias de intervención y de políticas educativas.

En tal sentido, un importante cuerpo de investigaciones se ha centrado en el estudio de aquellas variables que juegan un papel importante en el desempeño académico, mostrando la incidencia que sobre éste tienen los factores motivacionales (Yu y Patterson, 2010; Aguilar, Valencia y Martínez, 2004; Richardson, Abraham y Bond, 2012). 
Frente a los factores motivacionales se ha encontrado que la autoeficacia, la morosidad y el temor al fracaso se relacionan de manera significativa con el grado de cumplimiento de las actividades escolares y con el desempeño académico de los estudiantes (Aguilar, Valencia y Martínez, 2004; Vargas, 2007; Yu y Patterson, 2010; Navarro, 2003; Migues, Uzquiano y Lozano, 2010).

En la revisión empírica se ha concluido que la autoeficacia predice de manera significativa el desempeño académico, en la medida en que los estudiantes que se perciben como competentes presentan un incremento sustancial en la motivación intrínseca y son diligentes en sus actividades. Por el contrario, los estudiantes que se perciben como incapaces presentan disminución de su motivación, mayor morosidad y bajo desempeño académico (Pintrich y García, 1993; Suldo y Shaffer, 2008; Adeyemo, 2007; Beteta, 2008).

De otra parte, se ha encontrado que los estudiantes que presentan altos niveles de morosidad o procrastinación (Balkis y Duru, 2009; Balkis, 2013) y temor al fracaso tienden a presentar menor desempeño académico, en parte, relacionado con altos niveles de ansiedad y baja motivación (Lay y Silverman, 1996; Steel, 2007; Rosario et al., 2009).

Particularmente en la adolescencia, los estudiantes tienden a presentar problemas de autoeficacia al enfrentarse a cambios a nivel biológico, psicológico y social, los cuales pueden percibirse como abrumadores (Bandura, Pastorelli, Barbaranelli y Caprara, 1999). Adicionalmente, es una etapa en la que se procrastina el estudio de exámenes y el desarrollo de actividades académicas, en parte porque los adolescentes temen fracasar y no superar las expectativas y exigencias de sus padres, generándose un sentido de frustración, baja autoestima y bajo desempeño académico (Contreras et al., 2011; Pychyl, Coplan y Reid, 2002).

De esta manera, cobra relevancia estudiar estos aspectos en el contexto colombiano para tener una base empírica sobre los factores motivacionales y el desempeño académico en adolescentes que permita el diseño de estrategias específicas de intervención en esta población. 
Por lo tanto, en este estudio se exploró si los factores motivacionales (morosidad, temor al fracaso y autoeficacia) se relacionan con el desempeño académico en adolescentes colombianos y de qué manera se establece esta relación.

\section{Método}

Participaron 326 estudiantes, 53.68\% mujeres y $46.31 \%$ hombres, pertenecientes a cuatro instituciones educativas no oficiales de la ciudad de Tunja. La edad de los participantes osciló entre 11 y 18 años, con una edad promedio de 13.48 y D.E. de 1.68.

La muestra se estableció a través de un muestreo probabilístico multietápico por conglomerados con afijación proporcional por colegio y grado. Se emplearon los instrumentos Modelo de las interrelaciones entre disposiciones motivacionales generales y específicas, de Aguilar, Valencia y Martínez (2004) y el reporte de calificaciones de los estudiantes obtenidas en los dos periodos académicos en las áreas de lenguaje, ciencias y matemáticas.

\section{Resultados}

A partir de un análisis de regresión lineal múltiple se encontró que los factores motivacionales predicen $6 \%$ de la varianza de desempeño académico. En el modelo, la variable problemas de autoeficacia y la edad como predictor de control resultaron ser estadísticamente significativos. Las variables morosidad y el temor al fracaso no resultaron asociarse con el desempeño académico.

Los resultados indican que por cada unidad logarítmica en desempeño académico el predictor problemas de autoeficacia ( $\mathrm{t}=-3.717, \mathrm{p}=.000$ ) en -.259 [IC. - $.039,-.012$ ] y la edad $(\mathrm{t}=-2.207, \mathrm{p}=.028)$ en -.125 [IC. -.141, -.008] decrecen significativamente. Es decir, que los estudiantes que presentan mejor desempeño académico tienden a presentar menos problemas de autoeficacia y los estudiantes más jóvenes presentan mejor desempeño académico. 
La correlación semiparcial del modelo de regresión indicó que los predictores por sí mismos (por ejemplo, controlando el efecto de los demás predictores) explican la varianza del desempeño académico en porcentajes pequeños. Así pues, la autoeficacia da cuenta de $4 \%$ y la edad de $1.4 \%$.

Por su parte, el análisis descriptivo arrojó que las puntuaciones medias obtenidas por los estudiantes en los predictores incluidos en el modelo se ubican en niveles promedio, es decir, que aunque los estudiantes presenten algunas dificultades en cada uno de los factores evaluados (morosidad, temor al fracaso y autoeficacia) no alcanzan indicadores clínicos.

\section{Conclusiones}

La autoeficacia resulta ser un predictor significativo del desempeño académico, de tal manera que tener una percepción de competencia frente a las actividades escolares y frente a sí mismo conduce a presentar un mejor desempeño académico (Aguilar, Valencia y Martínez, 2004; Contreras et al., 2005; Galicia-Moyeda, Sánchez-Velasco y Robles-Ojeda, 2013).

Contrario a otros estudios, los factores de "morosidad" y "temor al fracaso" no resultaron ser significativos para explicar el desempeño académico (Aguilar, Valencia y Martínez, 2004; Balkis, y Duru, 2009; Balkis, 2013); sin embargo, no podrían arrojarse conclusiones definitivas en cuanto a que la población objeto de estudio presentó desempeño académico básico y satisfactorio y las puntuaciones obtenidas por los estudiantes en estos factores se ubican en rangos normales. Sería importante analizar la relación existente de estas variables cuando los estudiantes tengan desempeños bajos y deficientes y altos niveles de morosidad y temor al fracaso.

Finalmente, la autoeficacia es un factor fundamental en el desempeño académico, de tal manera que es importante trabajar en el fortalecimiento de la autoeficacia académica en los adolescentes. 


\section{Referencias}

Aguilar, J., Valencia, A. y Martínez, M. (2002). Un modelo de las interrelaciones entre disposiciones motivacionales generales y específicas (119-127). Revista Latina de Pensamiento $y$ Lenguaje, 10.

Adeyemo, D. (2007). Moderating Influence of Emotional Intelligence on the Link Between Academic Self-efficacy and Achievement of University Students (199-213). Psychology Developing Societies, 19.

Bandura, A., Pastorelli, C., Barbaranelli, C. y Caprara, G.V. (1999). Self-Efficacy Pathways to Childhood Depression (258-269). Journal of Personality and Social Psychology, 76.

Balkis, M. y Duru, E. (2009). Prevalence of Academic Procrastination Behavior Among Preservice Teachers and its Relationships with Demographics and Individual Preferences (18-32). Egitimde Kuram ve Uygulama, 5.

Balkis, M. (2013). Academic Procrastination, Academic Life Satisfaction and Academic Achievement: The Mediation Role of Rational Beliefs About Studying (57-74). Journal of Cognitive and Behavioral Psychotherapies, 13.

Beteta, M.C. (2008). Relación entre la autoeficacia y el rendimiento escolar y los hábitos de estudio en alumnos de secundaria (59-99). Revista Iberoamericana sobre Calidad, Eficacia y Cambio en Educación, 6.

Contreras, F., Espinosa, J., Esguerra, G., Haikal, A., Polanía, A. y Rodríguez, A. (2005). Autoeficacia, ansiedad y rendimiento académico en adolescentes (183-194). Diversitas: Perspectivas en Psicología, 1.

Contreras, H., Mori, E., Lam, N., Gil, E., Hinostroza, W., Rojas, D., Espinoza, E., Torrejón, E. y Conspira, C. (2011). Procrastinación en el estudio: exploración del fenómeno en adolescentes escolarizados (1-5). Revista Peruana de Epidemiología, 15.

Galicia-Moyeda, I.X., Sánchez-Velasco, A. y Robles-Ojeda, F.J. (2013). Autoeficacia en escolares adolescentes: su relación 
con la depresión, el rendimiento académico y las relaciones familiares (491-500). Anales de Psicología, 29.

Jiménez, M. (2000). Competencia social: intervención preventiva en la escuela (21-48). Infancia y Sociedad, 24.

Lay, C. y Silverman, S. (1996). Trait Procrastination, Anxiety, and Dilatory Behavior (61-67). Personality and Individual Differences, 21. doi: 10.1016/0191-8869(96)00038-4.

Migues, A.R., Uzquiano, M.P. y Lozano, A.B. (2010). Cambios en las variables predictoras del rendimiento escolar en enseñanza secundaria (790-796). Psicothema, 22.

Navarro, R. (2003). El rendimiento académico: concepto, investigación y desarrollo (1-15). Revista electrónica Iberoamericana sobre Calidad, Eficacia y Cambio en la Educación, 1. Pintrich, P.R. y García, T. (1993). Intraindividual Differences in Students, Motivation and Selfregulated Learning (99-107). German Journal of Educational Psichology, 7.

Pychyl, T.A., Coplan, R.J. y Reid, P.A. (2002). Parenting and Procrastination: Gender Differences in the Relations Between Procrastination, Parenting Style and Self-Worth in Early Adolescence (271-285). Personality and Individual Differences, 33.

Richardson, M., Abraham, C. y Bond, R. (2012). Psychological Correlates of University Students' Academic Performance: A Systematic Review and Meta-Analysis (353-387). Psychological Bulletin, 138. doi: 0.1037/a0026838.

Rosario, P., Costa, M., Núñez, J.C., González-Pienda, J., Solano, P. y Valle, A. (2009). Academic Procrastination: Associations with Personal, School, and Family Variables (118-127). The Spanish Journal of Psychology, 12.

Steel, P. (2007). The Nature of Procrastination: A Meta-Analytic and Theoretical Review of Quintessential Self-Regulatory Failure (65-94). Psychological Bulletin, 133. doi: 10.1037/0033-2909.133.1.65.

Suldo, S.M. y Shaffer, E.J. (2008). Looking Beyond Psychopathology: The Dual-Factor Model of Mental Health in Youth (52-68). School Psychology Review, 37. 
Vargas, G.M.G. (2007). Factores asociados al rendimiento académico en estudiantes universitarios, una reflexión desde la calidad de la educación superior pública (43-63). Revista Educación, 31.

Yu, F. y Patterson, D. (2010). Examining Adolescent Academic Achievement: A Cross-Cultural Review (324-327). The Family Journal, 18. doi: 10.1177/1066480710372071. 
